\title{
Effects of Korean Red Ginseng on Dry Mouth: A Randomized, Double- Blind, Placebo-Controlled Trial
}

\author{
Jae-Woo Park ${ }^{1 *}$, Beom-Joon Lee ${ }^{2}$, Youngmin Bu ${ }^{3}$, Inkwon Yeo ${ }^{4}$, Jinsung Kim ${ }^{1}$, and Bongha Ryu ${ }^{1}$ \\ ${ }^{1}$ Department of Internal Medicine, College of Oriental Medicine, Kyung Hee University, Seoul 130-701, Korea \\ ${ }^{2}$ Department of Internal Medicine, Kangnam Korean Hospital, Kyung Hee University, Seoul 135-501, Korea \\ ${ }^{3}$ Department of Herbal Pharmacology, College of Oriental Medicine, Kyung Hee University, Seoul 130-701, Korea \\ ${ }^{4}$ Department of Statistics, Sookmyung Women's University, Seoul 140-742, Korea
}

Dry mouth is easily neglected if not associated with oral diseases. Consequently, xerostomatic patients often use unconventional therapies. In traditional Korean medicine, Korean red ginseng (KRG) has long been used to relieve dry mouth. However, no clinical trials have investigated whether KRG actually has an effect on dry mouth. This study was performed to evaluate the efficacy of KRG for dry mouth. We enrolled 100 volunteers with no obvious oral or salivary gland diseases and divided them into KRG and placebo groups. Each group was divided into six subgroups according to age and gender. The subjects received $6 \mathrm{~g}$ /day of KRG or placebo for 8 weeks. The dry mouth visual analog scale (VAS), salivary flow rate, and a dry mouth-related symptom questionnaire were evaluated at baseline and at 4 and 8 weeks. KRG treatment did not show any significant differences for any of the variables. However, KRG improved the dry mouth VAS at 4 weeks and dry mouthrelated symptoms at 8 weeks in women, but not in men. Subgroup analyses revealed that KRG markedly improved the dry mouth VAS in women of menopausal age (40 to 59 years) at 4 and 8 weeks. KRG may have beneficial effects for dry mouth in women, especially those of menopausal age, but not in men. Further investigation in post- and perimenopausal women is required to elaborate on these findings.

Keywords: Dry mouth, Korean red ginseng, Traditional Korean medicine, Salivary flow rate, Pain measurement

\section{INTRODUCTION}

Dry mouth is a subjective feeling of oral dryness that occurs in $46 \%$ of the elderly population [1,2]. It is closely related to hyposalivation and occurs as a side effect of medications and diseases, such as Sjögren's syndrome and radiation-induced xerostomia [3]. In diseaseor radiation-induced dry mouth, the symptoms tend to be very severe and may lower the patient's quality of life and increase the risk of opportunistic oral infections [4]. In contrast, dry mouth unrelated to severe systemic and salivary gland diseases is easily neglected despite its high prevalence [1].

Therefore, many studies have focused on agents that are believed to relieve dry mouth or enhance salivary function, such as pilocarpine, sugarless gum, moisturizing gels or sprays, and artificial saliva substitutes [5-8]. Although these agents may increase salivary function, this does not always seem to improve the symptoms of dry mouth $[9,10]$. Natural products or traditional folk medicines have been regarded as other sources of curative agents for dry mouth. For example, some herbal (c) This is an Open Access article distributed under the terms of the Creative Commons Attribution Non-Commercial License (http://creativecommons.org/licenses/by-nc/3.0/) which permits unrestricted non-commercial use, distribution, and reproduction in any medium, provided the original work is properly cited.
Received 16 Mar. 2010, Revised 25 May 2010, Accepted 26 May 2010

*Corresponding author

E-mail: pjw2907@khu.ac.kr

Tel: +82-2-440-6219, Fax: +82-2-440-6295 
formulas, homeopathic medicines, ophiopogoins, and linseed extract have been studied for this purpose [11-15].

Korean red ginseng (KRG), the steamed root of Panax ginseng C.A. Meyer, is one of the best-known herbs and has been reported to have tonic effects on the immune system, sexual function, and the health of postmenopausal women [16-21]. In traditional Korean medicine, KRG has long been used to relieve the symptoms of dry mouth. However, no clinical trials have investigated whether KRG has an effect on this condition. Therefore, this study was performed to examine the effects of KRG on dry mouth. For this purpose, before and after administering KRG to xerostomatic patients for 8 weeks, we evaluated the dry mouth visual analog scale (VAS), unstimulated salivary flow rate (USFR), and stimulated salivary flow rate (SSFR), and the sub- jects completed a dry mouth-related symptom questionnaire. This study also investigated age and gender as factors contributing to dry mouth.

\section{MATERIALS AND METHODS}

\section{Subjects}

From September 2007 to May 2008, 133 volunteers were enrolled in the trial. Thirty-three of the subjects (24.8\%, 18 women [13.5\%] and 15 men [11.3\%]) were excluded (Fig. 1). All measurements and interviews were conducted at the East-West Neo-Medical Center, Kyung Hee University, Seoul, Korea.

The existence of subjective oral dryness and dry mouth (VAS $\geq 4$ ) at the screening visit served as an inclusion criterion [14]. The exclusion criteria were as follows:

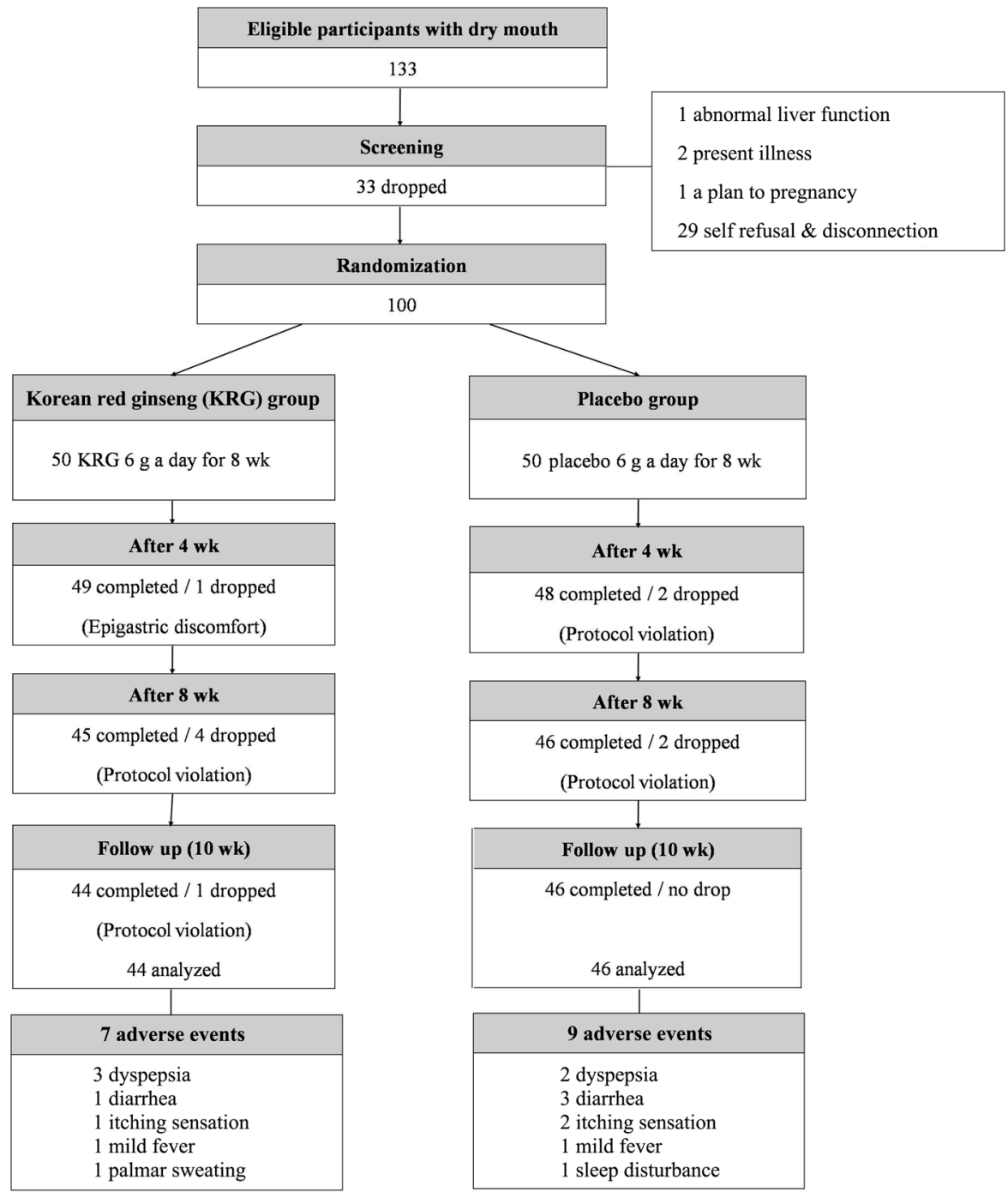

Fig. 1. Flow chart of the trial. 
Sjögren's syndrome, a history of irradiation therapy, severe psychiatric diseases, intake of herbal formulas up to 2 weeks before participation, ongoing treatment for dry mouth, and pregnancy or breastfeeding.

The study protocol was approved by the Institutional Review Board of East-West Neo-Medical Center, Kyung Hee University. Informed consent was obtained from all subjects prior to the study.

\section{Study design and assessment}

This randomized, double-blind, placebo-controlled, parallel-group trial was designed to investigate the effects of KRG on dry mouth. At the first visit, the dry mouth VAS and questionnaire were administered, and saliva was collected. The subjects were then stratified into subsets according to xerogenic drug use [3]. Subjects were assigned randomly in blocks of four using a computer random number generator to receive either 6 $\mathrm{g}$ of KRG (Korean Red Ginseng Powder Capsule ${ }^{\circledR}$; Korea Ginseng Corporation, Daejeon, Korea) or placebo (a capsule containing cornstarch powder with KRG flavoring) daily for 8 weeks. A voucher specimen was deposited with Korea Ginseng Corporation. The randomization list was kept by an independent pharmacist responsible for the KRG distribution until the end of the study. The subjects were given a diary, which they were asked to fill in twice a day after taking KRG or placebo.

On the second visit (4 weeks), dry mouth VAS and samples of saliva were evaluated. On the third visit (8 weeks), the three tests from the first visit were repeated. During each visit, the diaries were checked and the remaining capsules were counted. Compliance was estimated as a percentage of the number of capsules taken $v s$. the number of capsules that should have been taken. At 10 weeks, dry mouth VAS and side effects were recorded for each subject during a telephone call.

\section{Evaluation of dry mouth and questionnaire}

A global improvement in dry mouth was the primary outcome in this study. The subjects were asked to compare their overall dry mouth status on a 100-mM VAS at 4,8 , and 10 weeks $v s$. their status at baseline. The anchor points of the VAS were no dry mouth (0) and severe dry mouth (100). At baseline and 8 weeks, a modified six-item dry mouth-related symptom questionnaire study using a VAS [22] was administered (Table 1).

\section{Estimation of USFR and SSFR}

The USFR and SSFR were measured, at baseline and at 4 and 8 weeks, during rest and then during me-
Table 1. Six-item dry mouth-related symptom questionnaire

1. Does your mouth feel dry at night or on awakening (Dry-NA)?

2. Does your mouth feel dry at other times of the day (Dry-Day)?

3. Does your mouth feel dry when eating a meal (Dry-Eat)?

4. Do you have difficulties swallowing any foods (Dif-Swal)?

5. Does the amount of saliva in your mouth seem to be too little, too much, or you don't notice it (Am-Sal)?

6. How much does the dry mouth symptom interfere with the overall aspects of your life (Eff-Life)?

Subjects rated the difficulty they experienced due to dry mouth during the previous week.

The anchor points of the visual analog scale were no difficulty at all (0 $\mathrm{mM}$ ) and great difficulty $(100 \mathrm{mM})$.

chanical stimulation by chewing Parafilm (American National Can, Greenwich, CT, USA). The saliva was collected between 08:30 and 10:30. Before the first visit, the subjects were instructed not to eat, drink, or use any form of tobacco for 2 hours before saliva collection and to relax for about 10 minutes before the test. Unstimulated and Parafilm-stimulated saliva samples were collected for 5 minutes in preweighed disposable cups. During the first 5-minute period, saliva was expectorated at 30-second intervals, and the flow rate $(\mathrm{mL} / \mathrm{min})$ was calculated as the USFR. During the next 5-minute period, the subject was given Parafilm and instructed to chew it at a constant rate of 1 chew per second. The Parafilm-stimulated saliva flow rate $(\mathrm{mL} / \mathrm{min})$ was used as the SSFR. The saliva secreted during the first 30 seconds of each collection was discarded. Each salivary flow rate was determined by gravitation, using a scale with an accuracy of $0.01 \mathrm{~g}$ (AR2130 Adventurer Balance; Ohaus Pine Brook, NJ, USA), assuming that 1 $\mathrm{g}$ of saliva is equivalent to $1 \mathrm{~mL}$.

\section{Data analysis}

The efficacy data, such as the dry mouth VAS, USFR, SSFR, and each item or the sum totals of the questionnaire, were evaluated by per-protocol analysis, because only the pre- and posttreatment changes were considered.

For additional analysis of the contributing factors, the KRG and placebo groups were subdivided into two groups according to gender (women and men) and then into three subgroups according to age [23]. Therefore, the final subgroups were as follows: men's subgroups A to $\mathrm{C}$ (age $\leq 39,40$ to 59 , and $\geq 60$ years) and women's subgroups A to $\mathrm{C}$ (same age intervals).

\section{Statistical analysis}

The differences among all of the variables were as- 
sessed using the repeated $t$-test, linear mixed model, independent $t$-test, or the Mann-Whitney $U$-test. The demographic variables (age, baseline dry mouth VAS, baseline USFR, and SSFR) were analyzed by independent $t$-test. Gender, medications, dentures, and menopausal status at baseline were assessed using the $\chi^{2}$-test. Statistical analyses were performed using SPSS ver. 14.0 (SPSS Inc., Chicago, IL, USA). In all analyses, $p<0.05$ was taken to indicate statistical significance.

\section{RESULTS}

\section{Subject characteristics and baseline values}

Of the 100 subjects included in this trial (age, 19 to 76 years), 90 (90\%) completed the protocol. The mean compliance was $94 \%$ (95\% CI, 89 to 99) for both groups. During the study, one man withdrew from the placebo group owing to dyspepsia, and nine subjects (one woman and four men in the KRG group; three women and one man in the placebo group) dropped out because of delayed visits or insufficient KRG consumption (Fig. 1). The patient characteristics and baseline values are shown in Table 2.

\section{Comparison of the dry mouth VAS between the KRG and placebo groups}

Compared with the baseline, the mean values of the dry mouth VAS decreased throughout the study in both groups. However, there were no significant differences between the KRG and placebo groups at 4,8 , and 10 weeks (Table 3).

\section{Comparison of USFR and SSFR between the KRG and placebo groups}

Compared with the baseline, the mean USFR and SSFR increased throughout the study in both groups. However, there were no significant differences between the two groups at 4 and 8 weeks (Table 3 ).

\section{Comparison of dry mouth-related symptom ques- tionnaire between the KRG and placebo groups}

Both groups reported improvements in each item and showed improved total score of the questionnaire survey at 8 weeks. However, the KRG group showed no significant improvement in any item or the total score compared with the placebo group (Table 4).

Table 2. Baseline characteristics of subjects in KRG and placebo groups

\begin{tabular}{lccc}
\hline \multicolumn{1}{c}{ Characteristics } & $\begin{array}{c}\text { Placebo } \\
(n=50)\end{array}$ & $\begin{array}{c}\text { KRG } \\
(n=50)\end{array}$ & $p$-value \\
\hline Gender & $26(52)$ & $28(56)$ & 0.688 \\
$\quad$ Women & $24(48)$ & $22(44)$ & \\
$\quad$ Men & $12(46.2)$ & $15(53.8)$ & 0.586 \\
Menopausal women & $5(10)$ & $6(12)$ & 0.749 \\
Current dental dentures & $14(28)$ & $16(32)$ & 0.663 \\
Current medications & $11(22)$ & $10(20)$ & 0.806 \\
$\quad$ Antihypertensive & $2(4)$ & $1(2)$ & 0.558 \\
$\quad$ Hypoglycemic & $0(0)$ & $1(2)$ & 0.315 \\
Thrombolytic & $3(6)$ & $6(12)$ & 0.295 \\
Other ${ }^{1)}$ & $45.92 \pm 16.10$ & $48.28 \pm 15.82$ & 0.462 \\
Age (yr) & & & \\
Baseline values & $6.45 \pm 1.17$ & $6.85 \pm 1.32$ & 0.115 \\
Dry mouth VAS & $0.24 \pm 0.19$ & $0.20 \pm 0.14$ & 0.198 \\
USFR (mL/min) & $1.22 \pm 0.51$ & $1.05 \pm 0.62$ & 0.136 \\
SSFR (mL/min) &
\end{tabular}

Values are expressed as number (\%) or mean \pm SD

Gender, menopausal status, current denture wearing, and medications were analyzed by the $x^{2}$-test. Age, VAS, USFR, and SSFR were compared between the KRG and placebo groups using an independent $t$-test

KRG, Korean red ginseng; VAS, visual analogue scale; USFR, unstimulated salivary flow rate; SSFR, stimulated salivary flow rate

${ }^{1)}$ Other current medications denote antidiuretics, thyroid-related medications, and genitourinary medications

Table 3. Comparison of dry mouth VAS, USFR, and SSFR between KRG and placebo groups

\begin{tabular}{|c|c|c|c|c|}
\hline \multicolumn{2}{|c|}{ Variables } & $\begin{array}{c}\text { Placebo } \\
(n=46)\end{array}$ & $\begin{array}{c}\text { KRG } \\
(n=44)\end{array}$ & $p$-value \\
\hline \multirow{3}{*}{ Changes on dry mouth VAS } & $4 \mathrm{wk}$ - baseline & $-0.83 \pm 2.17$ & $-1.38 \pm 1.85$ & 0.198 \\
\hline & 8 wk - baseline & $-1.52 \pm 2.24$ & $-1.68 \pm 2.15$ & 0.727 \\
\hline & $10 \mathrm{wk}$ - baseline & $-1.76 \pm 2.51$ & $-1.84 \pm 2.03$ & 0.876 \\
\hline \multirow{2}{*}{ Changes on USFR } & 4 wk - baseline & $0.02 \pm 0.17$ & $0.04 \pm 0.10$ & 0.479 \\
\hline & 8 wk - baseline & $0.03 \pm 0.17$ & $0.01 \pm 0.11$ & 0.527 \\
\hline \multirow{2}{*}{ Changes on SSFR } & 4 wk - baseline & $0.08 \pm 0.39$ & $0.08 \pm 0.37$ & 0.967 \\
\hline & 8 wk - baseline & $0.14 \pm 0.49$ & $0.01 \pm 0.42$ & 0.165 \\
\hline
\end{tabular}

Values are expressed as mean $\pm S D$

KRG, Korean red ginseng; VAS, visual analogue scale; USFR, unstimulated salivary flow rate; SSFR, stimulated salivary flow rate 
Table 4. Comparison of six items and total scores of dry mouth-related symptom questionnaire between the KRG and placebo groups

\begin{tabular}{lccc}
\hline Item & $\begin{array}{c}\text { Placebo } \\
(n=46)\end{array}$ & $\begin{array}{c}\text { KRG } \\
(n=44)\end{array}$ & $p$-value \\
\hline Dry-NA & $-1.62 \pm 2.58$ & $-1.37 \pm 2.45$ & 0.467 \\
Dry-Day & $-1.13 \pm 2.11$ & $-1.46 \pm 1.93$ & 0.438 \\
Dry-Eat & $-0.62 \pm 2.14$ & $-1.18 \pm 2.11$ & 0.085 \\
Dif-Swal & $-0.86 \pm 2.27$ & $-1.14 \pm 2.06$ & 0.320 \\
Am-Sal & $-0.67 \pm 2.41$ & $-0.69 \pm 2.66$ & 0.731 \\
Eff-Life & $-1.34 \pm 2.17$ & $-1.49 \pm 2.62$ & 0.686 \\
Sum totals & $-6.24 \pm 10.98$ & $-7.34 \pm 9.99$ & 0.347
\end{tabular}

Values are expressed as means \pm SD. The dry mouth-related symptom questionnaire consists of six items on 100 -mM scales, where 0 indicates "no difficulty at all" and 100 indicates "great difficulty." Each value in this table represents the calculated value as the mean at 8 weeks minus the baseline

The meanings of the items are shown in Table 1. KRG, Korean red ginseng

\section{Comparison of the dry mouth VAS among sub- groups}

Time-related (baseline, 4, and 8 weeks) comparisons of the dry mouth VAS among the subgroups were assessed by repeated $t$-test and a linear mixed model. In comparisons among subgroups, no significant differences were observed using the repeated $t$-test (Figs. 2 and $3)$. However, the dry mouth VAS values in the KRG women's group and KRG women's subgroup B were significantly lower than those of the placebo women's group and placebo women's subgroup B, respectively $(p=0.047$ and $p=0.022$, respectively, with the linear

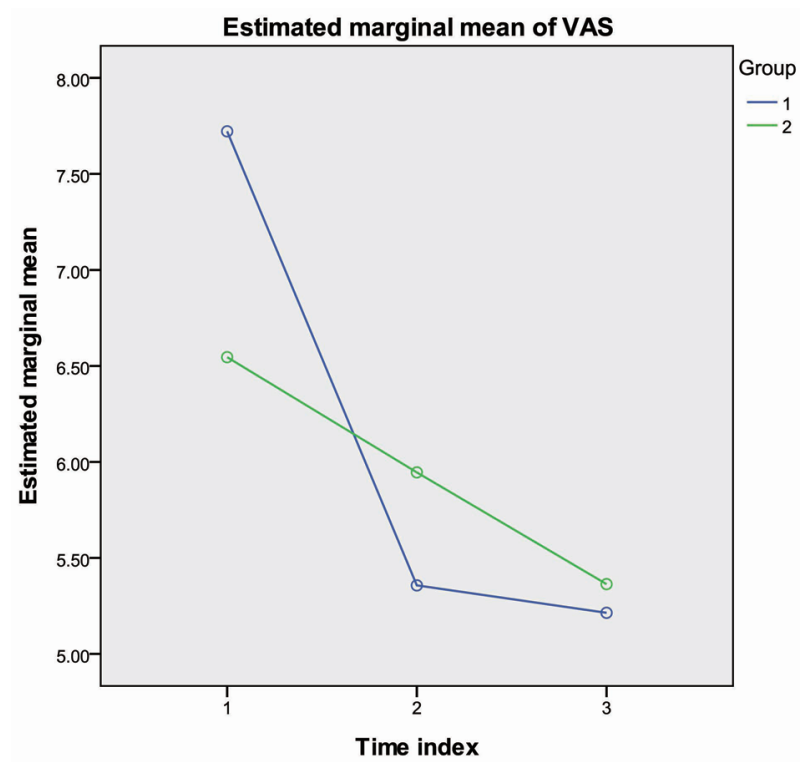

Fig. 2. Comparison on dry mouth visual analog scale (VAS) between women's groups by repeated $t$-test. 1, 2 and 3 in time index (horizontal axis) mean baseline, 4 and 8 weeks. Group 1, Korean red ginseng women's group (blue); group 2, placebo women's group (green). mixed model). For further statistical analyses, the changes in dry mouth VAS value between subgroups were compared. The changes in dry mouth VAS at 4 weeks in the KRG women's group and at 4 and 8 weeks in the KRG women's subgroup B were significantly greater than those in the placebo women's subgroup B $(p<0.05)$ (Table 5). However, the dry mouth VAS in these subgroups did not differ 2 weeks (follow-up period) after the treatments for 8 weeks. There were no significant changes among the other subgroups either (data not shown).

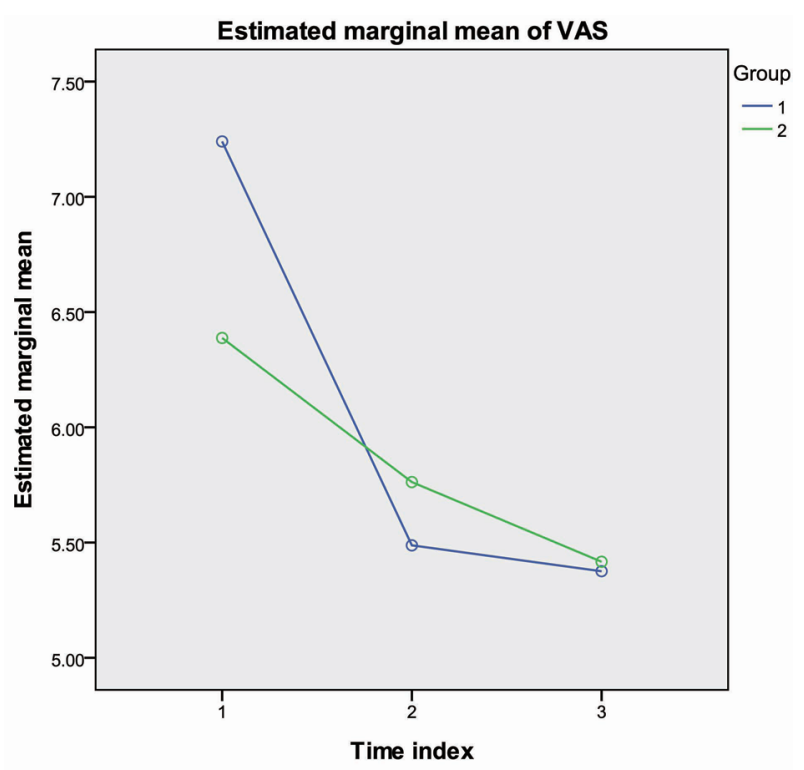

Fig. 3. Comparison on dry mouth visual analog scale (VAS) between women's subgroup B by repeated $t$-test. 1, 2 and 3 in time index (horizontal axis) mean baseline, 4 and 8 weeks. Group 1, Korean red ginseng women's subgroup B (blue); group 2, placebo women's subgroup B (green). 


\section{Comparison of USFR and SSFR among subgroups}

There were no significant changes in USFR and SSFR among the KRG and placebo subgroups (data not shown). There were also no significant differences in USFR and SSFR between the KRG group and placebo women's subgroup B.

\section{Comparison of the dry mouth-related symptom questionnaire among subgroups}

Time-related (baseline and 8 weeks) comparisons of the values of six items and total scores of the dry mouthrelated symptom questionnaire among the women's groups were assessed by repeated $t$-test (Table 6). All questionnaire values in the KRG women's group, with the exception of the item "Does the amount of saliva in your mouth seem to be too little, too much, or you don't notice it?" were lower than those of the placebo women's group. However, a significant improvement was seen in the item "How much does the dry mouth symptom interfere with the overall aspects of your life?" in the KRG women's group ( $p<0.05$, repeated $t$-test). There was no significant difference in dry mouth-related symptoms between the KRG women's subgroup B and placebo women's subgroup B.

\section{DISCUSSION}

This is the first trial to investigate the effects of KRG on patients with dry mouth who had no obvious oral diseases, such as Sjögren's syndrome or radiationinduced xerostomia. Age, gender, current medications, denture wearing, and menopausal status are known to be causative factors for dry mouth in the absence of organic oral diseases $[9,24]$. All of these factors were balanced at commencement of the trial. In additional analyses of subsets of subjects who had taken xerogenic medications, the baseline dry mouth VAS was $6.8 \pm 1.8$ in the KRG group $(n=16)$ vs. $6.2 \pm 1.2$ in the placebo group $(n=14)(p<0.21)$. Although the subject characteristics were balanced and the major oral diseases causing dry mouth were excluded, each group may have had heterogeneous features related to age and gender. Therefore, each group was divided into six subgroups according to age and gender [23].

The results showed that the dry mouth VAS, USFR, SSFR, and dry mouth-related symptom questionnaire items improved after 8 weeks compared with baseline. However, no significant differences were seen between values. Therefore, in contrast to our initial expectations, KRG may not improve dry mouth in subjects with no

Table 5. Comparison of changes on dry mouth VAS among subgroups in women

\begin{tabular}{|c|c|c|c|c|c|}
\hline \multirow{2}{*}{ Gender } & \multirow{2}{*}{$\begin{array}{l}\text { Age } \\
(\mathrm{yr})\end{array}$} & \multirow{2}{*}{ Group } & \multicolumn{3}{|c|}{ Changes on dry mouth VAS } \\
\hline & & & Baseline - 4 wk & Baseline - 8 wk & Baseline - $10 \mathrm{wk}$ \\
\hline \multirow{6}{*}{ Women } & & $\mathrm{KRG}(n=14)$ & $-2.36 \pm 1.89$ & $-2.51 \pm 1.63$ & $-2.87 \pm 2.17$ \\
\hline & $40-59$ & Placebo $(n=11)$ & $-0.55 \pm 2.50$ & $-1.27 \pm 2.10$ & $-1.73 \pm 3.10$ \\
\hline & & $p$-value & $0.037^{*}$ & $0.042^{*}$ & 0.228 \\
\hline & \multirow{3}{*}{ Total } & $\mathrm{KRG}(n=25)$ & $-1.75 \pm 1.56$ & $-1.86 \pm 2.32$ & $-2.02 \pm 2.31$ \\
\hline & & Placebo $(n=24)$ & $-0.63 \pm 2.02$ & $-1.08 \pm 1.95$ & $-1.75 \pm 2.54$ \\
\hline & & $p$-value & $0.044^{*}$ & 0.080 & 0.562 \\
\hline
\end{tabular}

Values are expressed as mean \pm SD

KRG, Korean red ginseng; VAS, visual analog scale

"Statistically significant by Mann-Whitney $U$-test $(p<0.05)$

Table 6. Comparison of each item and total score of dry mouth-related symptom questionnaire between the KRG and placebo women's groups

\begin{tabular}{lcccccc}
\hline & Dry-NA & Dry-Day & Dry-Eat & Dif-Swal & Am-Sal & Eff-Life \\
\hline Mean \pm SE & $-0.53 \pm 0.40$ & $-0.68 \pm 0.47$ & $-0.14 \pm 0.52$ & $-0.25 \pm 0.52$ & $0.32 \pm 0.50$ & $-0.92 \pm 0.41$ \\
Degree of freedom & 47 & 47 & 47 & 47 & 47 & 47 \\
T-statistic & -1.329 & -1.447 & -0.272 & -0.543 & -0.639 & -2.234 \\
$p$-value & 0.095 & 0.077 & 0.393 & 0.294 & 0.737 & $0.015^{*}$ \\
\hline
\end{tabular}

The questionnaire was assessed at baseline and 8 weeks

The meanings of each item are shown in Table 1. KRG, Korean red ginseng

*Statistically significant by repeated $t$-test $(p<0.05)$ 
history of organic oral disease.

In the subgroup analyses, KRG improved the dry mouth VAS in women, especially in women's subgroup B. One of the most important characteristics of this subgroup was post- and perimenopausal status, which is considered a key factor in the effect of KRG on dry mouth. Menopause is associated with a high prevalence of dry mouth [24,25], and indeed the proportion of postand perimenopausal women in each group in this study was high (Fig. 1). Generally, hormone replacement therapy (HRT) relieves oral discomfort, such as dry mouth and painful mouth symptoms, in menopausal women [26]; however, many menopausal women are concerned about the risk of breast cancer caused by HRT [27]. In contrast, the effects of KRG on menopausal symptoms and quality of life were not associated with any serious adverse effects in many of the earlier studies [16,28]. The mechanism of the effect of KRG on menopausal symptoms was not clear, but after 8 weeks of treatment with KRG, post- and perimenopausal women reported improvement in the dry mouth VAS. Examination of the subjects' medications showed that none of the women took HRT during the study, which may have affected dry mouth [16]. Moreover, KRG is also known to ameliorate climacteric symptoms by lowering blood levels of stress-related hormones, such as cortisol [16]. These findings suggested that KRG may be of benefit to menopausal women with subjective dry mouth who are not taking HRT.

In the present study, the salivary flow rates were not correlated with the dry mouth VAS in any of the groups, even in the KRG women's subgroup B (Table 3 ). These results were very similar to those of a previous study, which found no correlation between salivary flow rate and subjective dry mouth in xerostomatic patients without hyposalivation [29]. The higher baseline salivary flow rates may have affected the results in our study. In general, the criteria determining hyposalivation are USFR $<0.1 \mathrm{~mL} / \mathrm{min}$ and $\mathrm{SSFR}<1.0 \mathrm{~mL} / \mathrm{min}$ $[4,30]$, whereas the baseline USFR and SSFR in the present study were much higher for both groups (Table 2 ). Therefore, the effects of KRG on dry mouth in women's subgroup B may have been due to other factors, such as improvements in psychological status or hormonal changes [16], rather than a direct increase in salivary function. These results suggest that dry mouth may reflect other systemic conditions, rather than reduced salivary gland function [31].

In addition to an improvement in the dry mouth VAS, the KRG women's group also reported general improve- ments in most of the items and total scores of the questionnaire (Table 6). It was postulated that KRG may also have a positive effect on the quality of life associated with oral health as a tonic agent [28].

Many studies have suggested that different doses of KRG have diverse effects in a number of diseases, including erectile dysfunction, immunodeficiency, diabetes, and climacteric symptoms [16-21,32]. These studies suggested that a daily dose of 1 to $6 \mathrm{~g}$ of KRG for 12 weeks is safe in humans. Moreover, KRG has long been used in traditional Korean medicine as an important component of herbal formulas for the symptoms of dry mouth. As a result of these trials and in accordance with traditional Korean medical prescriptions, $6 \mathrm{~g}$ of $\mathrm{KRG}$ per day was set as the test dosage.

As in many KRG trials, our study indicated no serious adverse effects and no ginseng abuse syndrome. The subjects experienced only mild, intermittent discomfort, which was easily relieved at the end of the study. The efficacy of the blinding protocol was analyzed using self-suggestion, in which subjects were asked whether they believed the medications they had taken were real. Real KRG/placebo/uncertainty was 32 $(35.6 \%) / 11(12.2 \%) / 1(1.1 \%)$ in the KRG group and 25 $(27.8 \%) / 21(23.3 \%) / 0(0 \%)$ in the placebo group. From these results, we concluded that blinding was not broken $\left(p<0.084, \chi^{2}\right.$-test).

In conclusion, our results indicated that $6 \mathrm{~g}$ of $\mathrm{KRG}$ per day for 8 weeks may not have an obvious effect on subjective dry mouth, salivary flow rates, or dry mouthrelated quality of life in xerostomatic patients who have no history of oral diseases or hyposalivation. However, KRG improved subjective dry mouth in women, especially those aged 40 to 59 years, and dry mouth-related symptoms in women in general. Further investigation in post- and perimenopausal women is required to elaborate on these findings.

\section{ACKNOWLEDGEMENTS}

This work was supported by the 2007 grant from the Korean Society of Ginseng funded by Korea Ginseng Corporation.

\section{REFERENCES}

1. Sreebny LM, Valdini A. Xerostomia: a neglected symptom. Arch Intern Med 1987;147:1333-1337.

2. Narhi TO. Prevalence of subjective feelings of dry mouth in the elderly. J Dent Res 1994;73:20-25. 
3. Sreebny LM, Schwartz SS. A reference guide to drugs and dry mouth-2nd edition. Gerodontology 1997;14:33-47.

4. Torres SR, Peixoto CB, Caldas DM, Silva EB, Akiti T, Nucci M, de Uzeda M. Relationship between salivary flow rates and Candida counts in subjects with xerostomia. Oral Surg Oral Med Oral Pathol Oral Radiol Endod 2002;93:149-154.

5. Dodds MW, Hsieh SC, Johnson DA. The effect of increased mastication by daily gum-chewing on salivary gland output and dental plaque acidogenicity. J Dent Res 1991;70:1474-1478.

6. Samarawickrama DY. Saliva substitutes: how effective and safe are they? Oral Dis 2002;8:177-179.

7. Wu CH, Hsieh SC, Lee KL, Li KJ, Lu MC, Yu CL. Pilocarpine hydrochloride for the treatment of xerostomia in patients with Sjögren's syndrome in Taiwan-a double-blind, placebocontrolled trial. J Formos Med Assoc 2006;105:796-803.

8. Gil-Montoya JA, Guardia-López I, González-Moles MA. Evaluation of the clinical efficacy of a mouthwash and oral gel containing the antimicrobial proteins lactoperoxidase, lysozyme and lactoferrin in elderly patients with dry mouth-a pilot study. Gerodontology 2008;25:3-9.

9. Ship JA, Pillemer SR, Baum BJ. Xerostomia and the geriatric patient. J Am Geriatr Soc 2002;50:535-543.

10. Nagler RM. Salivary glands and the aging process: mechanistic aspects, health-status and medicinal-efficacy monitoring. Biogerontology 2004;5:223-233.

11. Kagami H, Horie K, Nishiguchi H, Shigetomi T, Ueda M. Effect of 'bakumondo-to', a Chinese-Japanese herbal medicine, on cultured and dispersed salivary gland cells. J Ethnopharmacol 1996;53:89-95.

12. Sugano S, Takeyama I, Ogino S, Kenmochi M, Kaneko T. Effectiveness of formula ophiopogoins in the treatment of xerostomia and pharyngoxerosis. Acta Otolaryngol Suppl 1996;522:124-129.

13. Johansson G, Andersson G, Edwardsson S, Bjorn AL, Manthorpe R, Attstrom R. Effects of mouthrinses with linseed extract Salinum without/with chlorhexidine on oral conditions in patients with Sjögren's syndrome: a double-blind crossover investigation. Gerodontology 2001;18:87-94.

14. Haila S, Koskinen A, Tenovuo J. Effects of homeopathic treatment on salivary flow rate and subjective symptoms in patients with oral dryness: a randomized trial. Homeopathy 2005;94:175-181.

15. Yanagi Y, Yasuda M, Hashida K, Kadokura Y, Yamamoto T, Suzaki H. Mechanism of salivary secretion enhancement by Byakkokaninjinto. Biol Pharm Bull 2008;31:431435.

16. Tode T, Kikuchi Y, Hirata J, Kita T, Nakata H, Nagata I. Effect of Korean red ginseng on psychological functions in patients with severe climacteric syndromes. Int J Gynaecol Obstet 1999;67:169-174.

17. Hong B, Ji YH, Hong JH, Nam KY, Ahn TY. A doubleblind crossover study evaluating the efficacy of korean red ginseng in patients with erectile dysfunction: a preliminary report. J Urol 2002;168:2070-2073.

18. Liou CJ, Huang WC, Tseng J. Long-term oral administration of ginseng extract modulates humoral immune response and spleen cell functions. Am J Chin Med 2005;33:651-661.

19. Lee BJ, Heo H, Oh SC, Lew JH. Comparison study of Korean and Chinese ginsengs on the regulation of lymphocyte proliferation and cytokine production. J Ginseng Res 2008;32:250-256.

20. Kim DI, Choi MS, Ahn HY. Efficacy and safety of red ginseng on women's health related quality of life and sexual function. J Ginseng Res 2009;33:115-126.

21. Shim MK, Lee YJ. Ginseng as a complementary and alternative medicine for postmenopausal symptoms. J Ginseng Res 2009;33:89-92.

22. Fox PC, Busch KA, Baum BJ. Subjective reports of xerostomia and objective measures of salivary gland performance. J Am Dent Assoc 1987;115:581-584.

23. Percival RS, Challacombe SJ, Marsh PD. Flow rates of resting whole and stimulated parotid saliva in relation to age and gender. J Dent Res 1994;73:1416-1420.

24. Frutos R, Rodriguez S, Miralles-Jorda L, Machuca G. Oral manifestations and dental treatment in menopause. Med Oral 2002;7:26-35.

25. Rivera Gomez B, Hernandez Vallejo G, Arriba de la Fuente L, Lopez Cantor M, Diaz M, Lopez Pintor RM. The relationship between the levels of salivary cortisol and the presence of xerostomia in menopausal women: a preliminary study. Med Oral Patol Oral Cir Bucal 2006;11:E407E412.

26. Forabosco A, Criscuolo M, Coukos G, Uccelli E, Weinstein R, Spinato S, Botticelli A, Volpe A. Efficacy of hormone replacement therapy in postmenopausal women with oral discomfort. Oral Surg Oral Med Oral Pathol 1992;73:570574.

27. Chen WY. Exogenous and endogenous hormones and breast cancer. Best Pract Res Clin Endocrinol Metab 2008;22:573585.

28. Ellis JM, Reddy P. Effects of Panax ginseng on quality of life. Ann Pharmacother 2002;36:375-379.

29. Kaplan I, Zuk-Paz L, Wolff A. Association between salivary flow rates, oral symptoms, and oral mucosal status. Oral Surg Oral Med Oral Pathol Oral Radiol Endod 2008;106:235-241.

30. Wolff M, Kleinberg I. Oral mucosal wetness in hypo- and normosalivators. Arch Oral Biol 1998;43:455-462. 
31. Atkinson JC, Grisius M, Massey W. Salivary hypofunction and xerostomia: diagnosis and treatment. Dent Clin North Am 2005;49:309-326.

32. Kim HY, Kang KS, Yamabe N, Yokozawa T. Comparison of the effects of Korean ginseng and heat-processed Korean ginseng on diabetic oxidative stress. Am J Chin Med 2008;36:989-1004. 